\title{
Atraumatic restorative techniques could reduce discomfort in children receiving dental treatment
}

\author{
Does the extent of discomfort differ between atraumatic restorative treatment \\ of multisurface cavities in deciduous molars compared with use of rotary \\ instruments?
}

\begin{abstract}
Schriks MCM, van Amerongen WE. Atraumatic perspective of ART: psychological and physiological aspects of treatment with and without rotary instruments. Community Dent Oral Epidemiol 2003; 31:15-20
\end{abstract}

Design This was a randomised controlled trial conducted in Indonesia.

Intervention Children, who each had at least one multisurface cavity in a deciduous molar, were randomly allocated to two treatment groups. The test group was treated using atraumatic restorative techniques (ART) using only hand instruments. The control group received treatment using rotary instruments (operated at $750 \mathrm{rpm}$ ), without water cooling. Glass ionomer cement was used for restoration in both groups.

Outcome measure The extent of discomfort was assessed by measuring the behavioural (psychological) and the physiological aspect represented by, respectively, a modified Venham score and the heart rate of the children at six fixed moments during dental treatment: (i) when the child entered the treatment room, (ii) at the start of excavation, (iii) at the moment of deepest excavation, (iv) at the moment of application of the matrix band and wedges, ( $v$ ) at the moment the restoration was applied, and (vi) after completion of the treatment.

Results A total of 403 children were treated. Venham scores showed a marked difference between the two groups at most timepoints. Heart rate measurements were different at deep excavation. Also, a clear relation between Venham scores and heart rate measurements could be found at all time points. Confounding could be shown for operating dentist, gender of the patient and initial anxiety, but not for age. No effect modification could be shown.

Conclusions Children treated according to the ART approach using hand instruments alone experience less discomfort than those treated using rotary instruments.

\section{Commentary}

ART were developed for use in developing countries, where access to conventional dental care is limited. The technique involves the use of hand instruments to access the carious lesion in primary teeth and remove the caries. The tooth is then restored with glass ionomer.

A number of trials have demonstrated that ART is an effective technique. The aim of this one was to compare ART with a minimal cavity preparation technique (MCP) utilising slow-speed handpieces and steel burs. The outcome measure was discomfort felt by the patient as assessed by alteration in patient heart rate and a trained observer using a behavioural assessment scale (the Modified Venham Index). ${ }^{1}$ It would have been interesting if the paper had also included a measure of baseline anxiety.

Four hundred and three children (of whom 202 had ART and 201 $\mathrm{MCP})$ had restorations placed. Interestingly, all children appear to have accepted some form of care, there being no record of procedures being abandoned. At all stages except baseline the Venham ratings were higher for the MCP group, suggesting more discomfort. This included at entry to the operating room (although not statistically significantly so), which is difficult to explain because the children where not aware of their treatment group at that point. Heart rate was again higher for the MCP group but only statistically significantly so at the time of the removal of the deepest caries. Perhaps not surprisingly, there was a significant operator effect, emphasising the effect of good behaviour management. Placement of the matrix band appears to cause discomfort, heart rate being highest for both ART and CMP at that time.

This study adds to the data indicating that ART is an appropriate restorative treatment technique. Dental caries is a significant problem across the world. It results in significant pain and sepsis for an unacceptably high number of children. Unfortunately, this problem is compounded by a lack of restorative care. Dentists in developed countries should consider ART as an appropriate technique and this study suggests that it is superior to restoration with handpieces. However, there are a number of further questions raised by this paper as the authors point out including, what would be the effect of well delivered local analgesia have been on the discomfort felt?

\section{Practice point}

- This study adds to the data indicating that ART is an appropriate restorative treatment technique.

\author{
Chris Deery \\ Paediatric Department, University of Edinburgh, Edinburgh \\ Dental Institute, Edinburgh, $U K$ \\ 1. Venham L, Bengston D, Cipes M. Children's response to sequential dental visits. \\ J Dent Res 1977:454-459.
}

\footnotetext{
Address for correspondence: MCM Schriks, Department of Cariology, Endodontology and Pedodontology, Academic Centre for Dentistry Amsterdam, Louwesweg 1, 1066 EA Amsterdam, The Netherlands. E-mail: m.schriks@acta.nl
}

Evidence-Based Dentistry (2005) 6, 9.

doi:10.1038/sj.ebd.6400310 